\title{
Benefits of aged garlic extract on Alzheimer's disease: Possible mechanisms of action (Review)
}

\author{
BUNGORN SRIPANIDKULCHAI \\ Center for Research and Development of Herbal Health Products, \\ Faculty of Pharmaceutical Sciences, Khon Kaen University, Khon Kaen 40002, Thailand
}

Received September 26, 2019; Accepted November 1, 2019

DOI: $10.3892 /$ etm. 2019.8390

\begin{abstract}
Alzheimer's disease (AD) is the most common form of dementia and has become a growing health concern in aging societies. $\beta$-amyloid $(A \beta)$ formation in vulnerable brain regions, such as the hippocampus and cerebral cortex is a major neuropathological feature of the disease. Currently, there is no specific drug available for the treatment of AD. However, due to its high antioxidant activity, aged garlic extract (AGE) has been widely used to prevent chronic diseases, such as cancer and cardiovascular disease. A number of studies on the benefits of AGE against cognitive and memory deficits have also been published. This review aimed to summarize the information related to the effects of AGE on learning memory in order to obtain a better understanding of its mechanisms of action. This review also presents an overview of the pathogenesis of $\mathrm{AD}$, and summarizes the main ingredients and neuroprotective effects of AGE against cognitive and learning memory deficits. The mechanisms of action of AGE are also discussed.
\end{abstract}

\section{Contents}

1. Alzheimer's disease

2. Aged garlic extract

3. Known neuroprotective effects of aged garlic extract

4. Known effects of aged garlic extract on learning memory

5. Overview of the mechanisms of action of aged garlic

6. Conclusions

Correspondence to: Dr Bungorn Sripanidkulchai, Center for Research and Development of Herbal Health Products, Faculty of Pharmaceutical Sciences, Khon Kaen University, Khon Kaen 40002, Thailand

E-mail: bungorn@kku.ac.th

Key words: Alzheimer's disease, aged garlic extract, cognitive and learning memory, neuroinflammation, antioxidant, cholinergic, glutamatergic, GABAergic

\section{Alzheimer's disease}

Alzheimer's disease (AD) is a progressive neurodegenerative disorder that commonly occurs among elderly with dementia (1). Currently, AD poses a serious threat to human health among the aging population. The disease is clinically diagnosed by a loss of cognitive function and memory, and pathologically by the deposition of senile plaque rich in the insoluble aggregation of $\beta$-amyloid $(A \beta)$ and neurofibrillary tangles in the brain $(2,3)$. The development of the disease is due to multiple factors, including both genetic and epigenetic factors. Diet, lifestyle and chemical exposure play important roles in the pathogenesis of the disease (4-6).

$\mathrm{A} \beta$ is a 40-42-amino acid residue of a transmembrane amyloid precursor protein that is cleaved by the enzymatic action of $\beta$-secretase and $\gamma$-secretase (7). Following polymerization, the $\mathrm{A} \beta$ oligomeric assemblies become a toxic molecule to form the deposited plaques, then activate the microglia and generate reactive oxygen species (ROS) and cytochemokines, leading to severe neuronal damage $(8,9)$. Several studies have demonstrated that the intra-cerebral injection of $A \beta$ causes neurodegeneration and an impairment of learning and memory $(10,11)$. A $\beta$ injection has been shown to induce radical-mediated neurotoxicity and an increase in lipid peroxidation. In animal models of $\mathrm{AD}, \mathrm{A} \beta$ binds to nicotinic acetylcholine receptors, which is abundantly expressed in the brain regions vulnerable to $\mathrm{AD}$, including the hippocampus and basal cholinergic forebrain (12-14). Apart from causing cholinergic degeneration, $A \beta$ leads to the disruption of signaling that is involved in long-term potentiation and memory in the hippocampus $(15,16)$.

Currently, there is no standard treatment or specific drug available for the treatment of AD. Drugs known to be associated with some parts of the pathogenesis and to reduce $A \beta$ formation by restoring cholinergic deficits, such as cholinesterase inhibitors (e.g., donepezil and rivastigmine) are used. Other drugs related to neuroinflammatory processes, such as cyclooxygenase-2 (COX-2) inhibitors (e.g., celebrex) and non-steroid anti-inflammatory drugs (NSAIDs; e.g., ibuprofen and indomethacin) are also used.

\section{Aged garlic extract}

Garlic (Allium sativum Linn., Alliaceae) has long been known, not only as a food ingredient, but also as a herbal 
medicine. Previous studies on garlic have emphasized its antioxidant properties against atherosclerosis and cancer (17-20). However, the consumption of raw garlic can cause various side-effects, including anemia, growth retardation and the destruction of the gut microflora, and can also alter serum protein levels $(21,22)$. Moreover, fresh garlic may cause indigestion and its pungent odor may linger on the breath, which may be a social barrier. To overcome these unwelcome effects, an alternative product from garlic that has less odor, but is rich in antioxidants has been produced and is known as aged garlic extract (AGE). AGE is an extract from garlic powder in a non-toxic solvent. It is derived from a prolonged extraction time at room temperature, generally $>15$ months. The aging process converts unstable compounds, such as allicin, to stable substances and several water-soluble organosulfur compounds, including the two major compounds, S-allylcysteine (SAC) and di-allyl-disulfide (DAD). AGE also contains other phytochemicals, such as ajoene, allixin, flavanoids, polyphenols and thiosulfinates $(23,24)$. Aged garlic extract has a very potent antioxidant activity, as well as numerous bio-activities, conferring several health benefits to humans (20). Recently, the potential activity of AGE to ameliorate cognitive impairment has attracted some interest.

\section{Known neuroprotective effects of aged garlic extract}

$\mathrm{A} \beta$ is known to exert cytotoxic effects on cultured neurons (25). There are multiple events culminating in neuronal death. The increased levels of ROS promote neuronal death $(26,27)$. Lipid peroxidation products are also increased in the brains of patients with $\mathrm{AD}$ (28). On the other hand, SAC, the major compound of AGE, has been shown to protect against the $\mathrm{A} \beta$ - and tunicamycin-induced death of differentiated PC12 cells (29).

$\mathrm{A} \beta$ induces neurotoxicity to hippocampal cells and increases the levels of ROS. A $\beta$ also induces the expression of the 78-kDa glucose-regulated protein (GRP-78), a molecular chaperone that regulates protein folding and translocation into the endoplasmic reticulum (ER), as well as the activation of caspase-12, which is localized predominantly at the ER $(30,31)$. SAC has been shown to decrease the levels of ROS and its neuroprotective effects are mediated predominantly through the caspase-12-dependent pathway in a concentration-dependent manner, but does not prevent the 4-hydroxynonenal-induced death of hippocampal neurons (32). These findings suggest that SAC exerts a unique effect on the ER and that it can protect against neuronal cell death that is triggered by ER dysfunction in the rat hippocampus. Moreover, a previous study on organotypic hippocampal slice cultures indicated that $A \beta$ potentiated the ER stress-induced neuronal death elicited by tunicamycin and that SAC exerted a significant neuroprotective effect against ER stress-induced neuronal death (33).

\section{Known effects of aged garlic extract on learning memory}

The attenuating effects of AGEon A $\beta$-induced neurotoxicity and cognitive impairment have been previously demonstrated (34). The ethyl acetate fraction of AGE reduces cellular oxidative stress, increases the viability and decreases the death of $\mathrm{PC} 12$ cells subjected to A $\beta$-induced cytotoxicity. Pre-treatment with

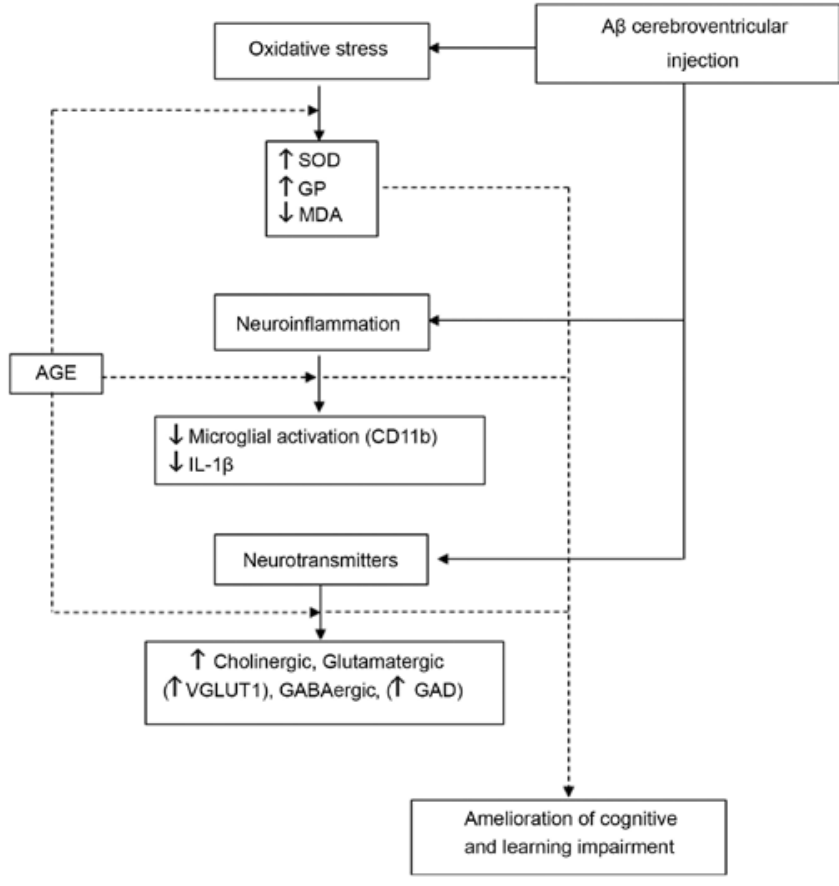

Figure 1. Possible mechanisms of action of AGE to ameliorate the cognitive impairment in rats with $A \beta$-induced neurotoxicity. AGE, aged garlic extract; $\mathrm{A} \beta, \beta$-amyloid; SOD, superoxide dismutase; GP, glutathione peroxidase; MDA, malondialdehyde; IL, interleukin; VGLUT1, vesicular glutamate transporter 1 protein; GAD, glutamate decarboxylase.

AGE has been shown to attenuate $A \beta$-induced learning and memory deficits in mice, as detected by the Y-maze test and a passive avoidance test. Dimethyl disulfide anhydrous has been identified to be a main thiosulfate of the ethyl acetate fraction of AGE that was obtained by subsequently partitioned of the ethanol extract of garlic at room temperature $\left(20^{\circ} \mathrm{C}\right)$. Experiments using transgenic mice have revealed the amelioration of early cognitive deficits by AGE (35). A comparative study was conducted in two models of transgenic mice, i.e., $\operatorname{Tg} 2576$ (slow plaque development with a predominance of $\mathrm{A} \beta$ 40) and TgCRND8 (accelerated plaque development with a predominance of $A \beta$ 42) (35). The feeding of AGE to the mice for 5 months was shown to prevent the deficits of spatial acquisition learning in both transgenic mouse models. AGE significantly reduced cerebral $A \beta$ build up and prevented the progressive deterioration of hippocampal-based cognitive tasks. In the senescence-accelerated mouse (SAM), which was established as a genetic murine model for studying spontaneous senescence (36), these mice have been characterized to be a prone strain in which the impairment of learning and memory with brain atrophy is accelerated (37). In addition, the chronic administration of AGE has been previously reported to prevent brain atrophy and improve learning memory, suggesting the anti-aging effects of AGE on SAM (38).

AGE has also been reported to prevent the lipopolysaccharide-induced cognitive deficits in rats via a reduction of lipid peroxidation markers, an increase in the activities of superoxide dismutase (SOD) and catalase, and an increase in the level of reduced glutathione, as well as a decrease in acetylcholinesterase activity, a downregulation in the expression of hippocampal nuclear factor- $\kappa \mathrm{B}$, Toll-like receptor 4 , glial fibrillary protein and interleukin (IL)-1 $\beta$ and an upregula- 
tion of nuclear factor (erythroid-derived 2)-like 2 (39). These findings suggest that AGE mitigates LPS-induced cognitive deficits and neuroinflammation, oxidative stress, astrogliosis and acethylcholinesterase activity.

\section{Overview of the mechanisms of action of aged garlic}

To better understand the beneficial effects of AGE, its mechanism of action should be clarified. However, as the pathogenesis of $\mathrm{AD}$ is complex (it is derived from both genetic and epigenetic factors), mechanisms of action of AGE on the improvement of cognitive and memory loss are also very complex. Clearly, its mechanisms of action are multifactorial and involve several pathways. In general, the high antioxidant activity of AGE is a critical element since there is evidence to support this. Our research group has previously reported that pre-treatment with AGE prevents the learning and short-term memory impairment caused by A $\beta$-induced neurotoxicity rats (40). In parallel, AGE can reverse neuronal loss in the CA1 and CA2 regions of the hippocampus, increase the activity of antioxidant enzymes, including SOD and glutathione peroxidase (GP), as well as decrease the malondialdehyde (MDA) level in the A $\beta$-exposed rat brain homogenate. Therefore, AGE, through its antioxidant activities, can scavenge free radicals through antioxidant enzyme activity to decrease the oxidative stress condition and help regain the spatial learning and memory performance in animal models with $\mathrm{A} \beta$-induced $\mathrm{AD}$.

The neurotoxicity of $A \beta$ has been reported to involve a number of neuronal events, such as the deficiency of neurotransmitters, and a decrease in vesicular transporters and synaptic transmissions, leading to neuronal cell death (41-44). In addition, the effects of AGE on cholinergic, glutamatergic and GABAergic systems have been shown in rats with $A \beta$-induced cognitive impairment (45). AGE can significantly improve the working memory, as well as the reference memory of rats with $A \beta$-induced cognitive impairment in association with the amelioration of the loss of cholinergic neurons. It also increases the vesicular glutamate transporter 1 protein (VGLUT1) and glutamate decarboxylase (GAD) levels in the hippocampus (45). These findings suggest that AGE attenuates the impairment of working memory via the modification of cholinergic neurons, VGLUT1 and GAD in the hippocampus of rats with $A \beta$-induced cognitive impairment. Since $A \beta$ formation, as a major neuropathological feature of $\mathrm{AD}$, has been shown to trigger a series of inflammatory processes; it can thus initiate the host defense response to damage neurons, contributing to neuronal degeneration $(46,47)$. Moreover, the activation of the microglia has been reported to promote the neurodegenerative process through the release of a number of pro-inflammatory cytokines that may lead to neuronal cell damage and eventual cell death (48-50). Based on these findings, the possible mechanisms of action of AGE on the process of neuroinflammation in $A \beta$-induced $A D$ have been proposed (51). It is noteworthy that pre-treatment with AGE significantly improves short-term recognition memory in rats with $A \beta$-induced neurotoxicity along with the reduction of the activation of microglia in the cerebral cortex and hippocampus via the decrease in the immunohistological staining of integrin aM or CD11b protein. Furthermore, the levels of IL-1 $\beta$ was also decreased in the hippocampus region (51). These studies suggest the possible roles of AGE as an antioxidant and anti-inflammatory agent. It is also suggested that it can act as a modulator of the activity and transportation of certain neurotransmitters to ameliorate the cognitive and learning memory deficits of $A \beta$-induced rat neurotoxicity (Fig. 1).

\section{Conclusions}

AGE is a potentially valuable product which can be used to prevent the deficits of cognitive and learning memory. In vitro and animal studies in vivo have demonstrated the mechanisms of action of AGE, which involve its antioxidant, anti-inflammatory and modulatory effects on neurotransmitter function in the brain regions that are associated with the pathogenesis of AD. However, as multiple factors are involved in the development of $\mathrm{AD}$, more pathways related to the disease pathogenesis need to be further investigated.

\section{Acknowledgements}

The author would like to thank Dr Suwannee Saenthaweesuk, all the co-authors of her publications on AGE and the authors of all the cited articles in this review. The stimulation to provide the review article from the organizing committee of International Aged Garlic Conference 2019 is also greatly appreciated.

\section{Funding}

No funding was received.

\section{Availability of data and materials}

Not applicable.

\section{Authors' contributions}

BS was responsible for the conception and design of this review article. BS also performed the literature search and edited and revised the article. The author has read and approved the final manuscript.

\section{Ethics approval and consent to participate}

Not applicable.

\section{Patient consent for publication}

Not applicable.

\section{Competing interests}

The author declares that there are no competing interests.

\section{References}

1. Alzheimer's Association: 2009 Alzheimer's disease facts and figures. Alzheimers Dement 5: 234-270, 2009.

2. Price DL, Sisodia SS and Borchelt DR: Genetic neurodegenerative diseases: The human illness and transgenic models. Science 282: 1079-1083, 1998. 
3. Varadarajan S, Yatin S, Aksenova M and Butterfield DA: Review: Alzheimer's amyloid beta-peptide-associated free radical oxidative stress and neurotoxicity. J Struct Biol 130: 184-208, 2000.

4. Lahiri DK, Maloney B and Zawia NH: The LEARn model: An epigenetic explanation for idiopathic neurobiological diseases. Mol Psychiatry 14: 992-1003, 2009.

5. Lahiri DK and Maloney B: The 'LEARn' (Latent Early-life Associated Regulation) model integrates environmental risk factors and the developmental basis of Alzheimer's disease, and proposes remedial steps. Exp Gerontol 45: 291-296, 2010.

6. Lahiri DK, Chen D, Ge YW, Bondy SC and Sharman EH Dietary supplementation with melatonin reduces levels of amyloid beta-peptides in the murine cerebral cortex. J Pineal Res 36: 224-231, 2004.

7. Vassar R, Kovacs DM, Yan R and Wong PC: The beta-secretase enzyme BACE in health and Alzheimer's disease: Regulation, cell biology, function, and therapeutic potential. J Neurosci 29 : 12787-12794, 2009

8. El Khoury J, Hickman SE, Thomas CA, Cao L, Silverstein SC and Loike JD: Scavenger receptor-mediated adhesion of microglia to beta-amyloid fibrils. Nature 382: 716-719, 1996.

9. Coraci IS, Husemann J, Berman JW, Hulette C, Dufour JH, Campanella GK, Luster AD, Silverstein SC and El-Khoury JB CD36, a class B scavenger receptor, is expressed on microglia in Alzheimer's disease brains and can mediate production of reactive oxygen species in response to beta-amyloid fibrils. Am J Pathol 160: 101-112, 2002.

10. Yamada K, Ren X and Nabeshima T: Perspectives of pharmacotherapy in Alzheimer's disease. Jpn J Pharmacol 80: 9-14, 1999.

11. Yamada K and Nabeshima T: Animal models of Alzheimer's disease and evaluation of anti-dementia drugs. Pharmacol Ther 88: 93-113, 2000.

12. Auld DS, Kornecook TJ, Bastianetto S and Quirion R: Alzheimer's disease and the basal forebrain cholinergic system: Relations to beta-amyloid peptides, cognition, and treatment strategies. Prog Neurobiol 68: 209-245, 2002

13. Dineley KT, Bell KA, Bui D and Sweatt JD: $\beta$-Amyloid peptide activates $\alpha 7$ nicotinic acetylcholine receptors expressed in Xenopus oocytes. J Biol Chem 277: 25056-25061, 2002.

14. Mesulam M, Shaw P, Mash D and Weintraub S: Cholinergic nucleus basalis tauopathy emerges early in the aging-MCI-AD continuum. Ann Neurol 55: 815-828, 2004.

15. Vitolo OV, Sant'Angelo A, Costanzo V, Battaglia F, Arancio O and Shelanski M: Amyloid beta -peptide inhibition of the PKA/CREB pathway and long-term potentiation: Reversibility by drugs that enhance cAMP signaling. Proc Natl Acad Sc USA 99: 13217-13221, 2002.

16. Balschun D, Wolfer DP, Gass P, Mantamadiotis T, Welzl H, Schütz G, Frey JU and Lipp HP: Does cAMP response element-binding protein have a pivotal role in hippocampal synaptic plasticity and hippocampus-dependent memory? J Neurosci 23: 6304-6314, 2003.

17. Ichikawa M, Ryu K, Yoshida J, Ide N, Yoshida S, Sasaoka T and Sumi S: Antioxidant effects of tetrahydro-beta-carboline derivatives identified in aged garlic extract. Biofactors 16: 57-72, 2002.

18. Durak I, Aytaç B, Atmaca Y, Devrim E, Avci A, Erol C and Oral D: Effects of garlic extract consumption on plasma and erythrocyte antioxidant parameters in atherosclerotic patients. Life Sci 75: 1959-1966, 2004.

19. Durak I, Kavutcu M, Aytaç B, Avci A, Devrim E, Ozbek H and Oztürk HS: Effects of garlic extract consumption on blood lipid and oxidant/antioxidant parameters in humans with high blood cholesterol. J Nutr Biochem 15: 373-377, 2004

20. Borek C: The health benefits of aged garlic extract. Townsend Lett Dr Patients 8-9: 112-116, 2004.

21. Nakagawa S, Masamoto K, Sumiyoshi H, Kunihiro K and Fuwa T: Effect of raw and extracted-aged garlic juice on growth of young rats and their organs after peroral administration (author's transl). J Toxicol Sci 5: 91-112, 1980 (In Japanese).

22. Shashikanth KN, Basappa SC and Sreenivasa Murthy V: A comparative study of raw garlic extract and tetracycline on caecal microflora and serum proteins of albino rats. Folia Microbiol (Praha) 29: 348-352, 1984

23. Amagase H, Petesch BL, Matsuura H, Kasuga S and Itakura $Y$ Intake of garlic and its bioactive components. J Nutr 131 (Suppl 3): 955S-962S, 2001

24. Chauhan NB: Multiplicity of garlic health effects and Alzheimer's disease. J Nutr Health Aging 9: 421-432, 2005.
25. Yao ZX, Drieu K, Szweda LI and Papadopoulos V: Free radicals and lipid peroxidation do not mediate beta-amyloid-induced neuronal cell death. Brain Res 847: 203-210, 1999.

26. Cassarino DS and Bennett JP Jr: An evaluation of the role of mitochondria in neurodegenerative diseases: Mitochondrial mutations and oxidative pathology, protective nuclear responses, and cell death in neurodegeneration. Brain Res Brain Res Rev 29: $1-25,1999$.

27. Ishige K, Chen Q, Sagara Y and Schubert D: The activation of dopamine D4 receptors inhibits oxidative stress-induced nerve cell death. J Neurosci 21: 6069-6076, 2001

28. Miranda S, Opazo C, Larrondo LF, Muñoz FJ, Ruiz F, Leighton F and Inestrosa NC: The role of oxidative stress in the toxicity induced by amyloid $\beta$-peptide in Alzheimer's disease. Prog Neurobiol 62: 633-648, 2000

29. Ito $\mathrm{Y}$, Ito $\mathrm{M}$, Takagi $\mathrm{N}$, Saito $\mathrm{H}$ and Ishige K: Neurotoxicity induced by amyloid beta-peptide and ibotenic acid in organotypic hippocampal cultures: Protection by $S$-allyl-L-cysteine, a garlic compound. Brain Res 985: 98-107, 2003.

30. Yu Z, Luo H, Fu W and Mattson MP: The endoplasmic reticulum stress-responsive protein GRP78 protects neurons against excitotoxicity and apoptosis: Suppression of oxidative stress and stabilization of calcium homeostasis. Exp Neurol 155: 302-314, 1999.

31. Nakagawa T, Zhu H, Morishima N, Li E, Xu J, Yankner BA and Yuan J: Caspase-12 mediates endoplasmic-reticulum-specific apoptosis and cytotoxicity by amyloid- $\beta$. Nature 403: 98-103, 2000

32. Kosuge Y, Koen Y, Ishige K, Minami K, Urasawa H, Saito H and Ito Y: $S$-allyl-L-cysteine selectively protects cultured rat hippocampal neurons from amyloid beta-protein- and tunicamycin-induced neuronal death. Neuroscience 122: 885-895, 2003.

33. Imai T, Kosuge $Y$, Ishige $\mathrm{K}$ and Ito $\mathrm{Y}$ : Amyloid beta-protein potentiates tunicamycin-induced neuronal death in organotypic hippocampal slice cultures. Neuroscience 147: 639-651, 2007.

34. Jeong JH, Jeong HR, Jo YN, Kim HJ, Shin JH and Heo HJ: Ameliorating effects of aged garlic extracts against $A \beta$-induced neurotoxicity and cognitive impairment. BMC Complement Altern Med 13: 268, 2013

35. Chauhan NB and Sandoval J: Amelioration of early cognitive deficits by aged garlic extract in Alzheimer's transgenic mice. Phytother Res 21: 629-640, 2007.

36. Takeda T, Hosokawa M, Takeshita S, Irino M, Higuchi K, Matsushita T, Tomita Y, Yasuhira K, Hamamoto $\mathrm{H}$ and Shimizu K: A new murine model of accelerated senescence. Mech Ageing Dev 17: 183-194, 1981

37. Takeda T, Hosokawa M and Higuchi K: Senescence-accelerated mouse (SAM): A novel murine model of accelerated senescence. J Am Geriatr Soc 39: 911-919, 1991.

38. Nishiyama N, Moriguchi T and Saito H: Beneficial effects of aged garlic extract on learning and memory impairment in the senescence-accelerated mouse. Exp Gerontol 32: 149-160, 1997.

39. Zarezadeh $M$, Baluchnejadmojarad $T$, Kiasalari $Z$, Afshin-Majd S and Roghani M: Garlic active constituent s-allyl cysteine protects against lipopolysaccharide-induced cognitive deficits in the rat: Possible involved mechanisms. Eur J Pharmacol 795: 13-21, 2017.

40. Wichai1 T, Pannangrong W, Welbat JU, Chaichun A, Sripanidkulchai K and Sripanidkulchai B: Effects of aged garlic extract on spatial memory and oxidative damage in the brain of amyloid- $\beta$ induced rats. Songklanakarin J Sci Technol 41: 311-318, 2019

41. Shin K, Guo H, Cha Y, Ban YH, Seo W, Choi Y, Kim TS, Lee SP, Kim JC, Choi EK, et al: Cereboost ${ }^{\mathrm{TM}}$, an American ginseng extract, improves cognitive function via up-regulation of choline acetyltransferase expression and neuroprotection. Regul Toxicol Pharmacol 78: 53-58, 2016

42. Lim GP, Chu T, Yang F, Beech W, Frautschy SA and Cole GM: The curry spice curcumin reduces oxidative damage and amyloid pathology in an Alzheimer transgenic mouse. J Neurosci 21: 8370-8377, 2001

43. Canas PM,Simões AP, Rodrigues RJ and Cunha RA: Predominant loss of glutamatergic terminal markers in a $\beta$-amyloid peptide model of Alzheimer's disease. Neuropharmacology 76 (Pt A): 51-56, 2014.

44. Ray B, Chauhan NB and Lahiri DK: Oxidative insults to neurons and synapse are prevented by aged garlic extract and $S$-allyl-L-cysteine treatment in the neuronal culture and APP-Tg mouse model. J Neurochem 117: 388-402, 2011. 
45. Thorajak P, Pannangrong W, Welbat JU, Chaijaroonkhanarak W, Sripanidkulchai K and Sripanidkulchai B: Effects of Aged Garlic Extract on Cholinergic, Glutamatergic and GABAergic Systems with Regard to Cognitive Impairment in $A \beta$-Induced Rats. Nutrients 9: 686, 2017.

46. Akiyama H, Barger S, Barnum S, Bradt B, Bauer J, Cole GM, Cooper NR, Eikelenboom P, Emmerling M, Fiebich BL, et al: Inflammation and Alzheimer's disease. Neurobiol Aging 21: 383-421, 2000.

47. McGeer EG and McGeer PL: The importance of inflammatory mechanisms in Alzheimer disease. Exp Gerontol 33: 371-378, 1998.

48. Hickman SE, Allison EK and El Khoury J: Microglial dysfunction and defective beta-amyloid clearance pathways in aging Alzheimer's disease mice. J Neurosci 28: 8354-8360, 2008.

49. Johnston $\mathrm{H}$, Boutin $\mathrm{H}$ and Allan SM: Assessing the contribution of inflammation in models of Alzheimer's disease. Biochem Soc Trans 39: 886-890, 2011.
50. Rogers $\mathrm{J}$ and Lue LF: Microglial chemotaxis, activation, and phagocytosis of amyloid beta-peptide as linked phenomena in Alzheimer's disease. Neurochem Int 39: 333-340, 2001.

51. Nillert N, Pannangrong W, Welbat JU, Chaijaroonkhanarak W, Sripanidkulchai K and Sripanidkulchai B: Neuroprotective Effects of Aged Garlic Extract on Cognitive Dysfunction and Neuroinflammation Induced by $\beta$-Amyloid in Rats. Nutrients 9: 24, 2017.

This work is licensed under a Creative Commons Attribution-NonCommercial-NoDerivatives 4.0 International (CC BY-NC-ND 4.0) License. 